\title{
Detecting and sizing the width of vertical cracks on moving samples using laser spot thermography
}

\author{
by J. González ${ }^{*, \star *}$, A. Mendioroz* and A. Salazar*
}

\author{
* Departamento de Física Aplicada I, Escuela de Ingeniería de Bilbao, Universidad del País Vasco UPV/EHU, \\ Plaza Ingeniero Torres Quevedo 1, 48013 Bilbao, Spain, agustin.salazar@ehu.es \\ ** Department of Applied Physics, CINVESTAV Unidad Mérida, carretera Antigua a Progreso km6, A.P. 73 \\ Cordemex, Mérida Yucatán 97310, Mexico.
}

\begin{abstract}
We propose a method, based on laser spot thermography, to detect and size the width of vertical cracks on samples moving at constant speed by fitting the temperature profile along the line that contains the centre of the laser spot and is perpendicular to the crack to its analytical expression. This method is useful for in-line inspection in factories, for detecting and characterizing cracks in real time, without stopping the production chain. Experimental measurements on samples containing calibrated vertical cracks confirm the validity of the method to measure the crack width with high accuracy.
\end{abstract}

\section{Introduction}

The growing necessity of in-service non-destructive testing and evaluation of surface breaking cracks in a wide variety of devices is currently a challenging task for modern industries and laboratories. In the last decades, optically stimulated infrared thermography has gained attention because it is noncontact, safe and very sensitive to the presence of cracks. To detect this kind of defects, a focused laser beam is directed to the sample surface close to the crack: the crack produces a partial blockage of the heat flux, which results in an asymmetry in the surface temperature distribution.

In this work, we address the challenge of detecting and sizing the width of vertical cracks on samples moving at constant speed. This is the case of in-line production or in-line quality control processes in factories, where cracks must be detected in real time, without stopping the production chain. First, we have found an analytical expression for the surface temperature of a moving cracked sample when it is illuminated by a laser spot that remains at rest. Experimental measurements on stainless steel samples containing calibrated vertical cracks indicate that the method is very sensitive to detect cracks, even of submicronic width. Moreover, by fitting the temperature profiles to the analytical model, the width of the crack is obtained with high accuracy.

\section{Theory}

Figure 1 shows the cross section of a semi-infinite and opaque sample moving at constant speed $v$ to the left along the $x$-axis. The sample contains a surface breaking vertical crack of width $w$. It is illuminated by a CW laser of power $P_{o}$ of Gaussian profile focused to a radius $a$, whose centre remains at rest at the origin of coordinates. Adiabatic boundary conditions are assumed. We have obtained an analytical expression for the temperature profile along the $x$-axis at time $t$ by integrating the temperature contribution for each laser position since the laser was switched on at $-t_{0}$ until $t$ :

$$
\begin{aligned}
& T(x, 0,0, t)=\frac{2 P_{o}}{\varepsilon \sqrt{\pi^{3}}} \int_{-t_{o}}^{t} \frac{1}{\sqrt{t-\tau}} \frac{e^{-\frac{2[x+v(t-\tau)]^{2}}{a^{2}+8 D(t-\tau)}}}{a^{2}+8 D(t-\tau)} d \tau+\operatorname{sign}(x) \frac{P_{o}}{\varepsilon \pi^{2} a \sqrt{D}} \int_{-\infty}^{\infty} d x_{o}^{t} d \tau \frac{\operatorname{sign}\left(x_{o}\right)}{t-\tau} \frac{e^{-\frac{\left[x_{o}+v(t-\tau)\right]^{2}}{a^{2}}}-\frac{u^{2}}{4 D(t-\tau)}}{\sqrt{a^{2}+8 D(t-\tau)}} \times \\
& \times\left[1-\frac{\sqrt{4 \pi D(t-\tau)}}{K R_{t h}} \exp \left(\frac{\sqrt{4 \pi D(t-\tau)}}{K R_{t h}}-\frac{u}{\sqrt{4 \pi D(t-\tau)}}\right)^{2}\right] \operatorname{erfc}\left(\frac{\sqrt{4 D(t-\tau)}}{K R_{t h}}+\frac{u}{\sqrt{4 D(t-\tau)}}\right) \text {. }
\end{aligned}
$$

In particular, at $t=0$ the crack is at the origin of coordinates. In this equation $K, D$ and $\varepsilon$ are the thermal conductivity, diffusivity and effusivity respectively, $u=|x|+\left|x_{0}\right|$ and $R_{t h}$ is the thermal contact resistance of the crack, which is related to the crack width $w$ through the equation $R_{t h}=w / K_{\text {air }}$.

It is worth noting that, according to the relativity principle, by performing a Galilean transformation in Eq. (1) we can calculate the temperature profile in the complementary configuration, where the sample remains at rest while the laser is moving at constant speed along the $x$-axis to the right, the so-called Flying spot method. 


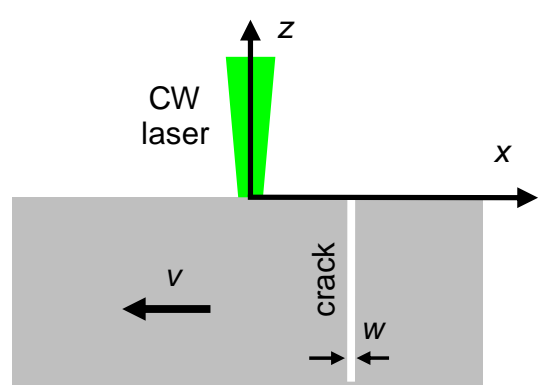

Fig. 1. Cross section of a sample containing a vertical crack and moving to the left at speed $v$. A CW laser is focused at the origin of coordinates.
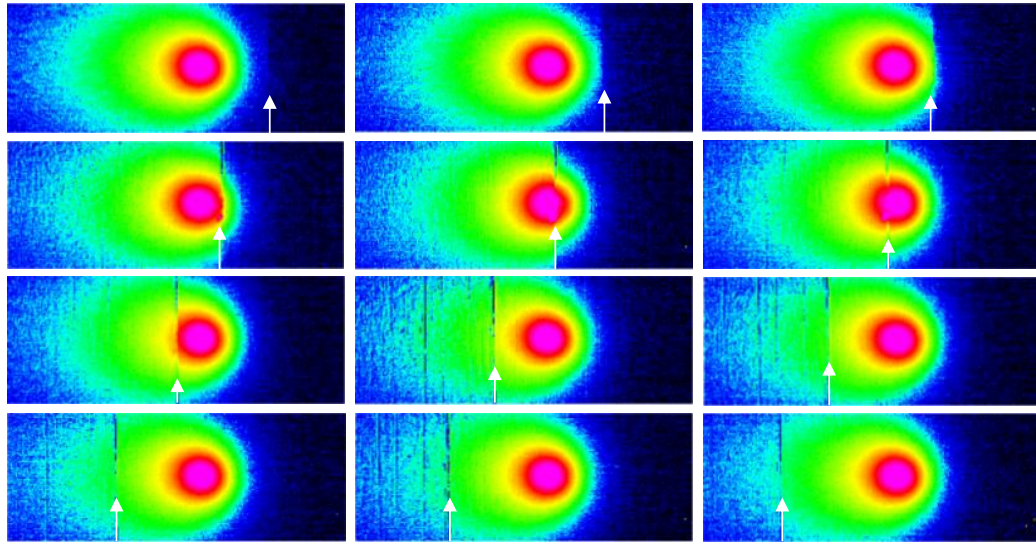

Fig. 2. Sequence of thermograms corresponding to a crack width $w=0.6$ $\mu \mathrm{m}$ in AISI-304. The laser is at rest while the sample is moving to the left at $v=3.56 \mathrm{~mm} / \mathrm{s}$. The arrow indicates the position of the crack.

\section{Experimental results and discussion}

To verify the validity of the proposed method we have prepared samples with calibrated cracks by putting in contact two AISI-304 blocks with nickel tapes of known thickness in between. Figure 2 shows a sequence of thermograms corresponding to a calibrated crack width $w=0.6 \mu \mathrm{m}$ when the sample is moving to the left at $v=3.56 \mathrm{~mm} / \mathrm{s}$. The white arrow indicates the position of the crack. Note that even for such a narrow fissure the temperature discontinuity is clearly visible.

In order to size the crack width we take the temperature profile along the horizontal line across the centre of the laser spot and perpendicular to the crack. Figures $3 a$ and $3 b$ show the profile corresponding to a position of the crack before reaching the laser for two sample speeds, 3.56 and $7.53 \mathrm{~mm} / \mathrm{s}$ respectively. Dots are the experimental data and the continuous lines the fits to Eq. (1) using a nonlinear least square fitting routine. Temperature profiles described by Eq. (1) depend on six parameters: $P_{o} / \varepsilon, K R_{t h}=K w / K_{\text {air }}, D, v, a$, and $t$, which governs the distance between the laser spot and the crack. The thermal properties of the sample $(D$ and $K)$ and the thermal conductivity of air $K_{\text {air }}$ are taken from the literature. Parameters $v$ and $t$ are measured very precisely from the IR video recorded by the camera due to the high acquisition frame rate (2000 images/s). The radius of the laser spot, $a$, is measured optically. Accordingly, the only parameters involved in the fittings of the temperature profiles are: $P_{o} / \varepsilon$ and $w$. The values in red in Fig. 3 correspond to the retrieved crack width. As can be observed the values are consistent and in good agreement with the nominal air gap widths. Fig. $3 c$ and $3 d$ are the same as the previous ones, but for a nominal crack width $w=2.7 \mu \mathrm{m}$. As before the agreement is very good, validating the method we are proposing in this work.
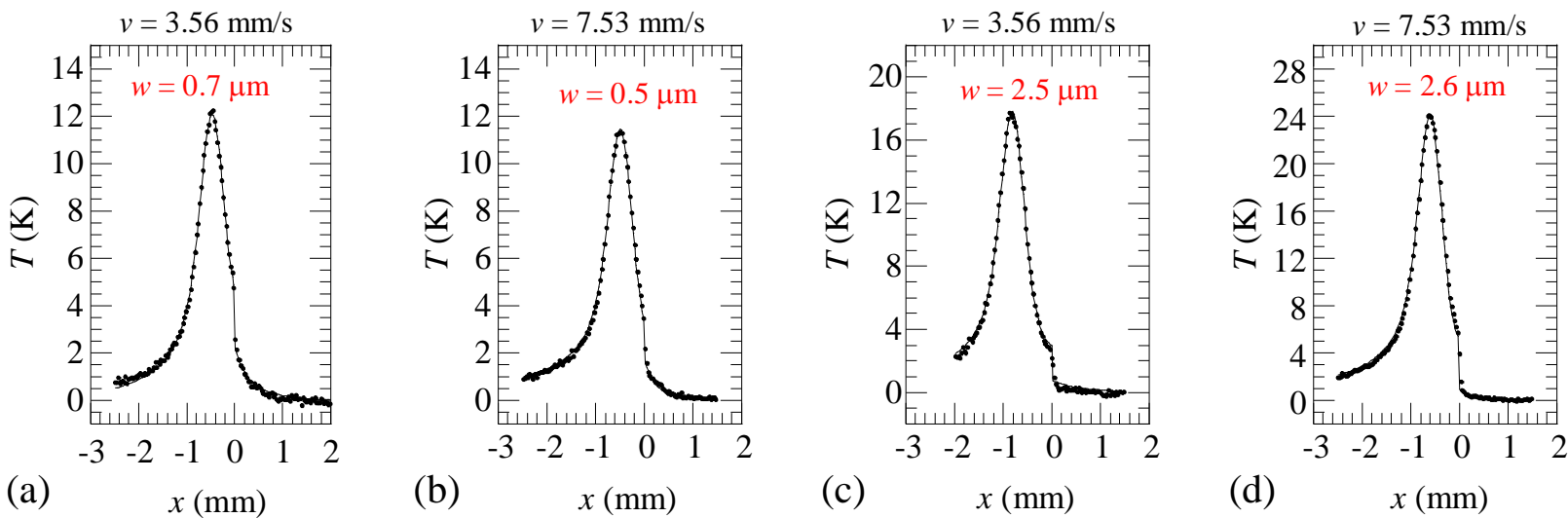

Fig. 3. Temperature profiles corresponding to two crack widths $w=0.6$ and $2.7 \mu m$, before reaching the laser. For each crack width two sample speeds are used. Dots are the experimental data and the continuous lines the fit to the model in Eq. (1). The retrieved crack widths are indicated in red.

This work has been supported by Ministerio de Economía y Competitividad, (DPI2016-77719-R, AEI/FEDER, UE), by Universidad del País Vasco UPV/EHU (GIU16/33) and by Gobierno Vasco (PIBA 2018-15). 